Some Applications of Fractional Calculus Involving Summation of Infinite Series

Susana Salinas de Romero and H.M. Srivastava 


\title{
Some Applications of Fractional Calculus Involving Summation of Infinite Series
}

\author{
Susana Salinas de Romero \\ Centro de Investigación de Matemática Aplicada \\ Facultad de Ingeniería \\ La Universidad del Zulia \\ Maracaibo, Venezuela \\ and \\ H.M. Srivastava \\ Department of Mathematics and Statistics \\ The University of Victoria \\ Victoria, British Columbia V8W $3 P 4$ \\ Canada
}

E-Mail: HMSRI@UVVM.UVIC.CA

\begin{abstract}
A significantly large number of earlier works on the subject of fractional calculus give interesting accounts of the theory and applications of fractional calculus operators in many different areas of mathematical analysis (such as ordinary and partial differential equations, integral equations, special functions, summation of series, et cetera). The main object of the present paper is to examine rather systematically (and extensively) some of the most recent contributions on the applications of fractional calculus operators in finding the sums of an interesting family of infinite series. Various further generalizations, relevant to the present investigation, are also given.

Key words and phrases. Fractional calculus, ordinary and partial differential equations, integral equations, special functions, summation of series, differintegral operator, Riemann-Liouville fractional derivative (or integral), Weyl fractional derivative (or integral), $N$-fractional calculus, Pochhammer symbol, Leibniz rule, hypergeometric functions, summation formula.
\end{abstract}

1991 Mathematics Subject Classification. Primary 26A33, 33C05; Secondary 33C20. 


\section{Introduction and Preliminaries}

The familiar differintegral operator ${ }_{c} D_{z}^{\mu}$ defined by

$$
{ }_{c} D_{z}^{\mu}\{f(z)\}:=\left\{\begin{array}{l}
\frac{1}{\Gamma(-\mu)} \int_{c}^{z}(z-\zeta)^{-\mu-1} f(\zeta) d \zeta(c \in \mathbb{R} ; \Re(\mu)<0) \\
\frac{d^{m}}{d z^{m}}{ }_{c} D_{z}^{\mu-m}\{f(z)\}(m-1 \leq \Re(\mu)<m ; m \in \mathbb{N}:=\{1,2,3, \cdots\}),
\end{array}\right.
$$

whenever the integral exists, happens to be one of the most frequently encountered tools in the theory of fractional calculus (that is, differentiation and integration of an arbitrary real or complex order). For $c=0$, the operator $D_{z}^{\mu}$ given by $[c f$. Equation (1)]

$$
D_{z}^{\mu}\{f(z)\}:={ }_{0} D_{z}^{\mu}\{f(z)\} \quad(\mu \in \mathbb{C})
$$

corresponds essentially to the classical Riemann-Liouville fractional derivative (or integral) of order $\mu$ (or $-\mu$ ). Furthermore, when $c \rightarrow \infty$, Equation (1) may easily be identified with the definition of the familiar Weyl fractional derivative (or integral) of order $\mu$ (or $-\mu$ ).

In a recent paper in the Journal of Fractional Calculus, Salinas de Romero et al. [1] made use of the $N$-fractional calculus of Nishimoto (cf., e.g., [2] and [3]) in order to prove an interesting summation formula which we choose to recall here in the following (corrected and slightly modified) form:

$$
\begin{aligned}
& \sum_{n=1}^{\infty}(-1)^{n-1}\left(\begin{array}{c}
\mu+n-1 \\
n
\end{array}\right) \sum_{k=0}^{\infty}\left(\begin{array}{l}
\mu \\
k
\end{array}\right) D_{z}^{-n-k}\{f(z)\} g(z) D_{z}^{n+k}\{h(z)\} \\
& +\sum_{n=1}^{\infty}(-1)^{n-1}\left(\begin{array}{c}
\mu+n-1 \\
n
\end{array}\right) \sum_{k=0}^{\infty}\left(\begin{array}{l}
\mu \\
k
\end{array}\right) \sum_{\ell=1}^{\infty}(-1)^{\ell}\left(\begin{array}{c}
n+k+\ell-1 \\
\ell
\end{array}\right) \\
& =\sum_{k=1}^{\infty}\left(\begin{array}{c}
\mu \\
k
\end{array}\right) D_{z}^{-n-k-\ell}\{f(z)\} D_{z}^{\ell}\{g(z)\} D_{z}^{n+k}\{h(z)\} \\
& +\sum_{k=1}^{\infty}\left(\begin{array}{c}
\mu \\
k
\end{array}\right) \sum_{\ell=1}^{\infty}(-1)^{\ell}\left(\begin{array}{c}
k+\ell-1 \\
\ell
\end{array}\right) D_{z}^{-k-\ell}\{f(z)\} D_{z}^{\ell}\{g(z)\} D_{z}^{k}\{h(z)\}
\end{aligned}
$$

where, and in what follows,

$$
\left(\begin{array}{l}
\mu \\
\kappa
\end{array}\right):=\frac{\Gamma(\mu+1)}{\Gamma(\mu-\kappa+1) \Gamma(\kappa+1)}=\left(\begin{array}{c}
\mu \\
\mu-\kappa
\end{array}\right) \quad(\mu, \kappa \in \mathbb{C}) .
$$


By setting $\mu=m(m \in \mathbb{N})$ and $g(z)=1$, and choosing the remaining functions $f(z)$ and $h(z)$ appropriately, Salinas de Romero et al. [1] also applied their main result (3) in order to derive the following summation formulas ( $c f .[1, \mathrm{pp} .40-41])$ :

$$
\begin{aligned}
& \sum_{n=1}^{\infty}(-1)^{n}\left(\begin{array}{c}
m+n-1 \\
n
\end{array}\right) \sum_{k=0}^{m}\left(\begin{array}{c}
m \\
k
\end{array}\right) \frac{\Gamma(-n-k-N-\beta)}{\Gamma(-N-\beta)}(a z)^{n+k} \\
& =-\sum_{k=1}^{m}\left(\begin{array}{c}
m \\
k
\end{array}\right) \frac{\Gamma(-k-N-\beta)}{\Gamma(-N-\beta)}(a z)^{k} \quad\left(N \in \mathbb{N}_{0}:=\mathbb{N} \cup\{0\}\right),
\end{aligned}
$$

which upon replacing $\beta$ by $\beta-N$ and $z$ by $(-z) / a$ yields the equivalent form:

$$
\begin{gathered}
\sum_{n=1}^{\infty}\left(\begin{array}{c}
m+n-1 \\
n
\end{array}\right) \sum_{k=0}^{m}\left(\begin{array}{c}
m \\
k
\end{array}\right) \Gamma(\beta+n+k+1) z^{n+k} \\
=-\sum_{k=1}^{m}\left(\begin{array}{c}
m \\
k
\end{array}\right) \Gamma(\beta+k+1) z^{k} ; \\
\sum_{n=1}^{\infty}\left(\begin{array}{c}
m+n-1 \\
n
\end{array}\right)(-a z)^{n} \sum_{k=0}^{m} \frac{\Gamma(\beta+N+1)}{\Gamma(\beta+n+k+N+1)}(a z)^{k} \cos \left(a z+\frac{\pi}{2}(n+k)\right) \\
=-\sum_{k=1}^{m}\left(\begin{array}{c}
m \\
k
\end{array}\right) \frac{\Gamma(\beta+N+1)}{\Gamma(\beta+k+N+1)}(-a z)^{k} \cos \left(a z+\frac{\pi}{2} k\right) \quad\left(N \in \mathbb{N}_{0}\right),
\end{gathered}
$$

which, upon replacing $\beta$ by $\beta-N$ and $z$ by $z / a$, yields the equivalent form:

$$
\begin{gathered}
\sum_{n=1}^{\infty}(-1)^{n-1}\left(\begin{array}{c}
m+n-1 \\
n
\end{array}\right) \sum_{k=0}^{m} \frac{z^{n+k}}{\Gamma(\beta+n+k+1)} \cos \left(z+\frac{\pi}{2}(n+k)\right) \\
=\sum_{k=1}^{m}\left(\begin{array}{c}
m \\
k
\end{array}\right) \frac{(-z)^{k}}{\Gamma(\beta+k+1)} \cos \left(z+\frac{\pi}{2} k\right) ; \\
\sum_{n=1}^{\infty}\left(\begin{array}{c}
m+n-1 \\
n
\end{array}\right)\left(-\frac{z}{1-z}\right)^{n} \sum_{k=0}^{m}\left(\begin{array}{c}
m \\
k
\end{array}\right) \frac{\Gamma(N) \Gamma(n+k-\beta+1)}{\Gamma(n+k+N) \Gamma(1-\beta)}\left(\frac{z}{1-z}\right)^{k} \\
=-\sum_{k=1}^{m}\left(\begin{array}{c}
m \\
k
\end{array}\right) \frac{\Gamma(N) \Gamma(k-\beta+1)}{\Gamma(k+N) \Gamma(1-\beta)}\left(\frac{z}{1-z}\right)^{k} \quad(z \neq 1 ; n \in \mathbb{N}),
\end{gathered}
$$


which, upon replacing $z$ by $z /(1+z)$, yields the equivalent form:

$$
\begin{gathered}
\sum_{n=1}^{\infty}(-1)^{n-1}\left(\begin{array}{c}
m+n-1 \\
n
\end{array}\right) \sum_{k=0}^{m}\left(\begin{array}{l}
m \\
k
\end{array}\right) \frac{\Gamma(n+k-\beta+1)}{\Gamma(n+k+N)} z^{k} \\
=\sum_{k=1}^{m}\left(\begin{array}{c}
m \\
k
\end{array}\right) \frac{\Gamma(k-\beta+1)}{\Gamma(1-\beta)} z^{k} \quad(N \in \mathbb{N}) ; \\
\sum_{n=1}^{\infty}\left(\begin{array}{c}
m+n-1 \\
n
\end{array}\right)\left(z^{2}\right)^{-n} \sum_{k=0}^{m}(-1)^{k}\left(\begin{array}{c}
m \\
k
\end{array}\right) \frac{\Gamma(n+k-\nu)}{\Gamma(\beta+n+k+1) \Gamma(-\nu)}\left(z^{2}\right)^{-k} \\
=-\sum_{k=1}^{m}\left(\begin{array}{c}
m \\
k
\end{array}\right) \frac{\Gamma(k-\nu)}{\Gamma(\beta+k+1) \Gamma(-\nu)}\left(z^{2}\right)^{-k} \quad(z \neq 0),
\end{gathered}
$$

which, upon replacing $z^{2}$ by $(-1) / z$ and multiplying both sides by $\Gamma(-\nu)$, yields the equivalent form:

$$
\begin{gathered}
\sum_{n=1}^{\infty}(-1)^{n-1}\left(\begin{array}{c}
m+n-1 \\
n
\end{array}\right) \sum_{k=0}^{m}\left(\begin{array}{c}
m \\
k
\end{array}\right) \frac{\Gamma(n+k-\nu)}{\Gamma(\beta+n+k+1)} z^{n+k} \\
=\sum_{k=1}^{m}\left(\begin{array}{c}
m \\
k
\end{array}\right) \frac{\Gamma(k-\nu)}{\Gamma(\beta+k+1)} z^{k} .
\end{gathered}
$$

It should be remarked in passing that each of the summation formulas (7), (9), and (11) appears erroneously in the work of Salinas de Romero et al. [1, p. 40, Equation (14); p. 41, Equations (15) and (16)]. More importantly, by setting $z=1$ in (5) and by (presumably) applying the Chu-Vandermonde theorem for summing a finite Gaussian hypergeometric ${ }_{2} F_{1}(1)$ series, Salinas de Romero et al. $[1$, p. 40, Equation (13)] claimed to have proved the summation formula:

$$
\begin{aligned}
\sum_{n=1}^{\infty}\left(\begin{array}{c}
m+n-1 \\
n
\end{array}\right) \frac{\Gamma(n) \Gamma(\beta+m+N+1)}{\Gamma(\beta+m+n+N+1)} a^{n} \\
=-\sum_{k=1}^{m}(-1)^{k}\left(\begin{array}{c}
m \\
k
\end{array}\right) \frac{\Gamma(\beta+N+1)}{\Gamma(\beta+k+N+1)} a^{k},
\end{aligned}
$$

which does not seem to hold true as stated above. As a matter of fact, by setting $z=1$ in 
(5), we actually get

$$
\begin{gathered}
\sum_{n=1}^{\infty}\left(\begin{array}{c}
m+n-1 \\
n
\end{array}\right) \frac{\Gamma(\beta+N+1)}{\Gamma(\beta+n+N+1)} a^{n}{ }_{1} F_{1}(-m ; \beta+n+N+1 ; a) \\
=-\sum_{k=1}^{m}(-1)^{k}\left(\begin{array}{c}
m \\
k
\end{array}\right) \frac{\Gamma(\beta+N+1)}{\Gamma(\beta+k+N+1)} a^{k},
\end{gathered}
$$

in which the aforementioned Chu-Vandermonde theorem cannot be applied to sum the finite (Kummer's) confluent hypergeometric ${ }_{1} F_{1}(a)$ series.

In the present paper we aim at investigating a unification (and generalization) of the summation formulas (5), (7), (9), and (11). We also consider the possibility of correcting (and, at the same time, extending) the erroneous result (13). Finally, we give a further generalization of the main summation formula (3) appearing in the work of Salinas de Romero et al. [1].

\section{A General Summation Theorem and Its Applications}

A closer examination of the summation formulas (5), (7), (9), and (11) [especially in their equivalent forms (6), (8), (10), and (12), respectively] would suggest their unification (as well as generalization) given by the following

Theorem. Let $\left\{\Omega_{n}\right\}_{n=0}^{\infty}$ be a suitably bounded sequence of complex numbers. Also let $\mu$ be a complex parameter.

Then

$$
\begin{gathered}
\sum_{n=1}^{\infty}(-1)^{n-1}\left(\begin{array}{c}
\mu+n-1 \\
n
\end{array}\right) \sum_{k=0}^{\infty}\left(\begin{array}{l}
\mu \\
k
\end{array}\right) \Omega_{n+k} \zeta^{n+k} \\
=\sum_{k=1}^{\infty}\left(\begin{array}{l}
\mu \\
k
\end{array}\right) \Omega_{k} \zeta^{k} \quad(\mu \in \mathbb{C}),
\end{gathered}
$$

provided further that the series involved converge absolutely.

Proof. For a direct proof of the general result (15), without using fractional calculus techniques, we denote by $\Xi(\mu ; \zeta)$ the first member of (15) and observe readily that

$$
\begin{aligned}
\Xi(\mu ; \zeta)=\sum_{k=0}^{\infty}\left(\begin{array}{c}
\mu \\
k
\end{array}\right) \Omega_{k} \zeta^{k} \\
\cdot\left\{1-{ }_{2} F_{1}(-k, \mu ; \mu-k+1 ; 1)\right\},
\end{aligned}
$$


where the inversion of the order of summation is justified by absolute convergence of the series involved.

Now, by the Chu-Vandermonde theorem (cf., e.g., Slater [4, p. 243, Equation (III.4)]), we have

$$
{ }_{2} F_{1}(-k, \mu ; \mu-k+1 ; 1)=\frac{(1-k)_{k}}{(\mu-k+1)_{k}}= \begin{cases}1 & (k=0) \\ 0 & (k \in \mathbb{N}),\end{cases}
$$

where, for convenience, $(\lambda)_{k}$ denotes the Pochhammer symbol defined by

$$
(\lambda)_{k}:=\frac{\Gamma(\lambda+k)}{\Gamma(\lambda)}= \begin{cases}1 & (k=0 ; \lambda \neq 0) \\ \lambda(\lambda+1) \cdots(\lambda+k-1) & (k \in \mathbb{N}) .\end{cases}
$$

Making use of (17) on the right-hand side of (16), we immediately obtain

$$
\Xi(\mu ; \zeta)=\sum_{k=1}^{\infty}\left(\begin{array}{l}
\mu \\
k
\end{array}\right) \Omega_{k} \zeta^{k},
$$

which is precisely the second member of the assertion (15). This evidently completes our direct proof of the Theorem under the various constraints stated already with (15).

Alternatively, by adding $\Omega_{0}$ to each member of (15) and then combining the series occurring on both sides of (15), we can easily rewrite the summation formula (15) in its equivalent form:

$$
\sum_{n=0}^{\infty}(-1)^{n}\left(\begin{array}{c}
\mu+n-1 \\
n
\end{array}\right) \sum_{k=0}^{\infty}\left(\begin{array}{l}
\mu \\
k
\end{array}\right) \Omega_{n+k} \zeta^{n+k}=\Omega_{0} \quad(\mu \in \mathbb{C})
$$

it being understood, as before, that the series involved converge absolutely.

Since

$$
\left(\begin{array}{l}
\mu \\
k
\end{array}\right)=\frac{(-1)^{k}(-\mu)_{k}}{k !} \quad\left(\mu \in \mathbb{C} ; k \in \mathbb{N}_{0}\right),
$$

this last summation formula (20) can be deduced from the following result of Chen and Srivastava $[5$, p...., Equation (3.12)]:

$$
\sum_{n=0}^{\infty}\left(\begin{array}{c}
\mu+n-1 \\
n
\end{array}\right) \sum_{k=0}^{\infty}(-\mu)_{k} \Delta_{n+k} \frac{\zeta^{n+k}}{k !}=\Delta_{0} \quad(\mu \in \mathbb{C}),
$$

by merely replacing $\Delta_{n}$ and $\zeta$ by $\Omega_{n}$ and $-\zeta$, respectively. 
The summation formulas (5), (7), (9), and (11) follow readily from the general result (15) when we set

(i) $\mu=m(m \in \mathbb{N}), \quad \Omega_{n}=\frac{\Gamma(-n-N-\beta)}{\Gamma(-N-\beta)}\left(n \in \mathbb{N}_{0}\right)$, and $\zeta=a z$;

(ii) $\mu=m(m \in \mathbb{N}), \quad \Omega_{n}=\frac{\Gamma(\beta+N+1)}{\Gamma(\beta+n+N+1)} \cos \left(a z+\frac{\pi}{2} n\right) \quad\left(n \in \mathbb{N}_{0}\right)$, and $\zeta=a z$;

(iii) $\mu=m(m \in \mathbb{N}), \quad \Omega_{n}=\frac{\Gamma(N) \Gamma(n-\beta+1)}{\Gamma(n+N) \Gamma(1-\beta)}\left(n \in \mathbb{N}_{0}\right)$, and $\zeta=\frac{z}{1-z}(z \neq 1)$; and

(iv) $\mu=m(m \in \mathbb{N}), \quad \Omega_{n}=\frac{\Gamma(n-\nu)}{\Gamma(\beta+n+1) \Gamma(-\nu)}\left(n \in \mathbb{N}_{0}\right)$, and $\zeta=\frac{1}{z^{2}}(z \neq 0)$, respectively.

\section{A Corrected Version of the Summation Formula (13)}

Keeping in view the familiar Gaussian summation theorem (cf., e.g., [4, p. 28, Equation $(1.7 .6)])$ :

$$
\begin{gathered}
{ }_{2} F_{1}(a, b ; c ; 1)=\frac{\Gamma(c) \Gamma(c-a-b)}{\Gamma(c-a) \Gamma(c-b)} \\
(\Re(c-a-b)>0 ; \quad c \neq 0,-1,-2, \cdots),
\end{gathered}
$$

we set

$$
\Omega_{n}=\frac{\Gamma(\alpha+n)}{\Gamma(\beta+n)} \quad\left(n \in \mathbb{N}_{0}\right) \quad \text { and } \quad \zeta=-1
$$

in (15), and we easily find that

$$
\begin{gathered}
\sum_{n=1}^{\infty}\left(\begin{array}{c}
\mu+n-1 \\
n
\end{array}\right) \frac{\Gamma(\alpha+n)}{\Gamma(\beta+n)}{ }_{2} F_{1}(-\mu, \alpha+n ; \beta+n ; 1) \\
=-\sum_{k=1}^{\infty}(-1)^{k}\left(\begin{array}{l}
\mu \\
k
\end{array}\right) \frac{\Gamma(\alpha+k)}{\Gamma(\beta+k)} .
\end{gathered}
$$

Now we apply (23) on the left-hand side of (24) and obtain the summation formula:

$$
\begin{aligned}
\sum_{n=1}^{\infty}\left(\begin{array}{c}
\mu+n-1 \\
n
\end{array}\right) \frac{\Gamma(\alpha+n) \Gamma(\beta+\mu-\alpha)}{\Gamma(\beta+\mu+n)} \\
=-\sum_{k=1}^{\infty}(-1)^{k}\left(\begin{array}{l}
\mu \\
k
\end{array}\right) \frac{\Gamma(\alpha+k) \Gamma(\beta-\alpha)}{\Gamma(\beta+k)}
\end{aligned}
$$

$(\mu \in \mathbb{C}: \quad \Re(\beta+\mu-\alpha)>0 ; \quad \beta \neq 0,-1,-2, \cdots)$. 
This last result (25) can indeed be verified directly by applying the Gaussian summation theorem (23) on both sides of (25).

A special case of (25) when

$$
\mu=m \quad(m \in \mathbb{N}), \quad \alpha=0, \quad \text { and } \quad \beta \rightarrow \beta+N+1
$$

yields the summation formula:

$$
\begin{aligned}
\sum_{n=1}^{\infty}\left(\begin{array}{c}
m+n-1 \\
n
\end{array}\right) \frac{\Gamma(n) \Gamma(\beta+m+N+1)}{\Gamma(\beta+m+n+N+1)} \\
=-\sum_{k=1}^{\infty}(-1)^{k}\left(\begin{array}{c}
m \\
k
\end{array}\right) \frac{\Gamma(k) \Gamma(\beta+N+1)}{\Gamma(\beta+k+N+1)}
\end{aligned}
$$

which provides the corrected version of (13).

In the mathematical literature there are many summation theorems [in addition, of course, to the Gaussian summation theorem (23) above] which hold true for various generalized hypergeometric ${ }_{p+1} F_{p}(x)$ series for special values of $p \in \mathbb{N}$ and $x \in \mathbb{R}$. Each of these known summation theorems (cf., e.g., Slater [4, pp. 243-246]) can be used, in a rather straightforward manner, in conjunction with our Theorem with a view to deducing from (15) a summation formula analogous to (25). The details involved in these derivations are fairly routine, and we choose to leave them as an exercise for the interested reader.

\section{Generalized Leibniz Rules and Their Applications}

The familiar Leibniz rule for ordinary derivatives admits itself of the following extension in terms of the Riemann-Liouville operator $D_{z}^{\mu}$ defined by (2):

$$
D_{z}^{\mu}\{f(z) g(z)\}=\sum_{n=0}^{\infty}\left(\begin{array}{l}
\mu \\
n
\end{array}\right) D_{z}^{\mu-n}\{f(z)\} D_{z}^{n}\{g(z)\} \quad(\mu \in \mathbb{C})
$$

which was applied iteratively by Salinas de Romero et al. [1] in order to prove their main result (3). 
The generalized Leibniz rule (27), which was also applied earlier by Galué et al. [6] in order to derive the summation identity:

$$
\begin{aligned}
\sum_{n=1}^{\infty}(-1)^{n-1} & \frac{(\mu)_{n}}{n !} \sum_{k=0}^{\infty}\left(\begin{array}{l}
\mu \\
k
\end{array}\right) D_{z}^{-n-k}\{f(z)\} D_{z}^{n+k}\{g(z)\} \\
= & \sum_{n=1}^{\infty}\left(\begin{array}{l}
\mu \\
n
\end{array}\right) D_{z}^{-n}\{f(z)\} D_{z}^{n}\{g(z)\} \quad(\mu \in \mathbb{C})
\end{aligned}
$$

suffers from an apparent drawback in the sense that the interchange of the functions $f(z)$ and $g(z)$ on the right-hand side is not obvious. (See also Galué [7] for several summation formulas [contained in the Chen-Srivastava sum (22)] which she deduced by suitably specializing the functions $f(z)$ and $g(z)$ in the summation identity (28) above.) A further (symmetrical) generalization of (27), considered by Watanabe [8] and Osler [9], without such a drawback, is given by (cf., e.g., Samko et al. [10, p. 316, Equation (17.12)])

$$
\begin{gathered}
D_{z}^{\mu}\{f(z) g(z)\}=\sum_{n=-\infty}^{\infty}\left(\begin{array}{c}
\mu \\
\sigma+n
\end{array}\right) D_{z}^{\mu-\sigma-n}\{f(z)\} D_{z}^{\sigma+n}\{g(z)\} \\
(\mu, \sigma \in \mathbb{C}),
\end{gathered}
$$

which, in the special case when $\sigma=0$, yields the Leibniz rule (27).

By applying the generalized Leibniz rule (29), Aular de Durán et al. [11] derived the following summation identity [11, p. 752, Equation (3.9)]:

$$
\begin{gathered}
\left(\begin{array}{c}
-\mu \\
\alpha
\end{array}\right) \sum_{\substack{n=-\infty \\
(n \neq 0)}}^{\infty}\left(\begin{array}{c}
\mu \\
\beta+n
\end{array}\right) D_{z}^{-\alpha-\beta-n}\{f(z)\} D_{z}^{\alpha+\beta+n}\{g(z)\} \\
=f(z) g(z)-\left(\begin{array}{c}
-\mu \\
\alpha
\end{array}\right)\left(\begin{array}{c}
\mu \\
\beta
\end{array}\right) D_{z}^{-\alpha-\beta}\{f(z)\} D_{z}^{\alpha+\beta}\{g(z)\} \\
-\sum_{\substack{n=-\infty \\
(n \neq 0)}}^{\infty}\left(\begin{array}{c}
-\mu \\
\alpha+n
\end{array}\right) \sum_{k=-\infty}^{\infty}\left(\begin{array}{c}
\mu \\
\gamma+k
\end{array}\right) D_{z}^{-\gamma-\alpha-n-k}\{f(z)\} \\
\cdot D_{z}^{\gamma+\alpha+n+k}\{g(z)\} \quad(\alpha, \beta, \gamma, \mu \in \mathbb{C}),
\end{gathered}
$$

which, in the special case when $\alpha=\beta=\gamma=0$, would reduce to the simpler summation identity (28). 
An obvious special case of the summation identity (30) when $\alpha=\beta=\rho$ happens to be the main result of Al-Zamel and Kalla [12, p. 30, Equation (5)], who also presented several examples illustrating the usefulness of this particular case of (30) in deriving various relationships involving infinite sums. The general summation identity (30), obtained earlier by Aular de Durán et al. [11], is potentially more advantageous in this respect than the aforementioned special case $(\alpha=\beta=\rho$ ) actually used by Al-Zamel and Kalla [12].

Now we turn to a mild extension of the generalized Leibniz rule (29) in the form ( $c f$., e.g., Samko et al. [10, p. 317]):

$$
\begin{gathered}
D_{z}^{\mu}\{f(z) g(z)\}=\sum_{n=-\infty}^{\infty} \kappa\left(\begin{array}{c}
\mu \\
\sigma+\kappa n
\end{array}\right) D_{z}^{\mu-\sigma-\kappa n}\{f(z)\} D_{z}^{\sigma+\kappa n}\{g(z)\} \\
(\mu, \sigma \in \mathbb{C} ; \quad 0<\kappa \leq 1),
\end{gathered}
$$

which, for $\kappa=1$, reduces at once to (29). Indeed, by applying this generalized Leibniz rule (31), Chen and Srivastava [5] derived the following summation identity [5, p..., Equation (5.12)]:

$$
\begin{aligned}
& \xi\left(\begin{array}{c}
-\mu \\
\alpha
\end{array}\right) \sum_{\substack{n=-\infty \\
(n \neq 0)}}^{\infty} \eta\left(\begin{array}{c}
\mu \\
\beta+\eta n
\end{array}\right) D_{z}^{-\alpha-\beta-\eta n}\{f(z)\} D_{z}^{\alpha+\beta+\eta n}\{g(z)\} \\
&=f(z) g(z)-\xi \eta\left(\begin{array}{c}
-\mu \\
\alpha
\end{array}\right)\left(\begin{array}{c}
\mu \\
\beta
\end{array}\right) D_{z}^{-\alpha-\beta}\{f(z)\} D_{z}^{\alpha+\beta}\{g(z)\} \\
&-\quad \sum_{\substack{n=-\infty \\
(n \neq 0)}}^{\infty} \xi\left(\begin{array}{c}
-\mu \\
\alpha+\xi n
\end{array}\right) \sum_{k=-\infty}^{\infty} \zeta\left(\begin{array}{c}
\mu \\
\gamma+\zeta k
\end{array}\right) D_{z}^{-\gamma-\alpha-\xi n-\zeta k}\{f(z)\} \\
& \cdot D_{z}^{\gamma+\alpha+\xi n+\zeta k}\{g(z)\} \quad(\alpha, \beta, \gamma, \mu \in \mathbb{C} ; \quad 0<\xi, \eta, \zeta \leq 1) .
\end{aligned}
$$

The summation identity (30), given earlier by Aular de Durán et al. [10], is an obvious special case of this last result (32) when

$$
\xi=\eta=\zeta=1 .
$$

Finally, if we apply the method of derivation of (3) mutatis mutandis, it is not difficult to make use of the generalized Leibniz rule (31) in order to deduce the following (presumably 
new) summation identity:

$$
\begin{aligned}
& \xi\left(\begin{array}{c}
-\mu \\
\alpha
\end{array}\right) \sum_{\substack{n=-\infty \\
(n \neq 0)}}^{\infty} \eta\left(\begin{array}{c}
\mu \\
\beta+\eta n
\end{array}\right) \sum_{k=-\infty}^{\infty} u\left(\begin{array}{c}
-\alpha-\beta-\eta n \\
\rho+u k
\end{array}\right) \\
& \cdot D_{z}^{-\alpha-\beta-\rho-\eta n-u k}\{f(z)\} D_{z}^{\rho+u k}\{g(z)\} D_{z}^{\alpha+\beta+\eta n}\{h(z)\} \\
& =f(z) g(z) h(z)-\xi \eta\left(\begin{array}{c}
-\mu \\
\alpha
\end{array}\right)\left(\begin{array}{l}
\mu \\
\beta
\end{array}\right) \sum_{n=-\infty}^{\infty} v\left(\begin{array}{c}
-\alpha-\beta \\
\lambda+v n
\end{array}\right) \\
& \text { - } D_{z}^{-\alpha-\beta-\lambda-v n}\{f(z)\} D_{z}^{\lambda+v n}\{g(z)\} D_{z}^{\alpha+\beta}\{h(z)\} \\
& -\sum_{\substack{n=-\infty \\
(n \neq 0)}}^{\infty} \xi\left(\begin{array}{c}
-\mu \\
\alpha+\xi n
\end{array}\right) \sum_{k=-\infty}^{\infty} \zeta\left(\begin{array}{c}
\mu \\
\gamma+\zeta k
\end{array}\right) \sum_{\ell=-\infty}^{\infty} w\left(\begin{array}{c}
-\gamma-\alpha-\xi n-\zeta k \\
\sigma+w \ell
\end{array}\right) \\
& \cdot D_{z}^{-\gamma-\alpha-\sigma-\xi n-\zeta k-w \ell}\{f(z)\} D_{z}^{\sigma+w \ell}\{g(z)\} \\
& \cdot D_{z}^{\gamma+\alpha+\xi n+\zeta k}\{h(z)\} \\
& (\alpha, \beta, \gamma, \lambda, \mu, \rho, \sigma \in \mathbb{C} ; \quad 0<\xi, \eta, \zeta, u, v, w \leq 1),
\end{aligned}
$$

provided that each member exists.

In its special case when $g(z)=1$ [and $h(z)$ is replaced by $g(z)$ ], if we further set

$$
\rho=\sigma=\lambda=0 \quad \text { and } \quad u=v=w=1
$$

the summation identity (33) would yield the earlier result (32) due to Aular de Durán et al. [11]. More importantly, upon setting

$$
\alpha=\beta=\gamma=\rho=\sigma=\lambda=0
$$

and

$$
\xi=\eta=\zeta=u=v=w=1
$$

the general summation identity (33) can be reduced fairly easily to the known result (3) given recently by Salinas de Romero et al. [1]. It should be remarked also that, in each of the general summation identities (30), (32), and (33), the second term on the righthand side corresponds obviously to the (excluded) term $n=0$ of the sum occurring on the left-hand side. 


\section{Acknowledgments}

The present investigation was initiated during the second-named author's visit to the Centro de Investigación de Matemática Aplicada (La Universidad del Zulia) at Maracaibo in July 1996. This work was supported, in part, by the Natural Sciences and Engineering Research Council of Canada under Grant OGP0007353. The first-named author thanks CONDES-La Universidad del Zulia for financial support.

\section{References}

[1] S. Salinas de Romero, S.L. Kalla, and S.B. Yakubovich, Applications of $N$-fractional calculus to infinite sums, J. Fractional Calculus 8(1995), 37-42.

[2] K. Nishimoto, Fractional Calculus, Vol. I, II, III, and IV, Descartes Press, Koriyama, 1984, 1987. 1989, and 1991.

[3] K. Nishimoto, An Essence of Nishimoto's Fractional Calculus (Calculus in the 21st Century): Integrations and Differentiations of Arbitrary Order, Descartes Press, Koriyama, 1991.

[4] L.J. Slater, Generalized Hypergeometric Functions, Cambridge University Press, Cambridge, London, and New York, 1966.

[5] M.-P. Chen and H.M. Srivastava, Fractional calculus operators and their applications involving power functions and summation of series, Appl. Math. Comput. 81(1997), 287-304.

[6] L. Galué, S.L. Kalla, and K. Nishimoto, Application of fractional calculus to infinite sums, J. Fractional Calculus 1(1992), 17-21.

[7] L. Galué, Application of fractional calculus to infinite sums (II), J. Fractional Calculus $7(1995), 61-67$.

[8] Y. Watanabe, Notes on the generalized derivative of Riemann-Liouville and its applications to Leibniz's formula. I and II, Tôhoku Math. J. 34(1931), 8-27 and 28-41.

[9] T.J. Osler, Leibniz rule for fractional derivatives generalized and an application to infinite series, SIAM J. Appl. Math. 18(1970), 658-674.

[10] S.G. Samko, A.A. Kilbas, and O.I. Marichev, Fractional Integrals and Derivatives: Theory and Applications, Gordon and Breach Science Publishers, Reading, Tokyo, Paris, Berlin, and Langhorne (Pennsylvania), 1993.

[11] J. Aular de Durán, S.L. Kalla, and H.M. Srivastava, Fractional calculus and the sums of certain families of infinite series, J. Math. Anal. Appl. 190(1995), 738-754.

[12] A. Al-Zamel and S. Kalla, An application of generalized Leibniz rule to infinite sums, J. Fractional Calculus 7(1995), 29-33. 